\title{
Fast and Easy Method of Automatic Camera Calibration
}

\author{
Jiang You ${ }^{a}$, Liwei Tang ${ }^{\mathrm{b}}$, Shijie Deng ${ }^{\mathrm{c}}$ \\ Department of Guns Engineering, Ordnance Engineering College, Shijiazhuang, 050003, China \\ ay_jiang1214@163.com, btom5171@163.com, 'dengshijie615171@163.com
}

Keywords: machine vision, automatic calibration, least square method, cross ratio invariability.

\begin{abstract}
The camera calibration with traditional methods is of low efficiency and big error, and it needs to extract boundary points manually. In order to improve the efficiency and automaticity degree of the camera calibration, a fast and easy method research of automatic camera calibration was presented. In this paper, we designed a new maker box with three circles of different radius to confirm the boundary and origin. And the detected the edge of the target circles by canny algorithm, and employed the least square method to match the center. Finally ranked the center dot matrix to match the 3D coordinate based the principle of cross ratio invariability, and then provided the results as the input of the Zhang's coplanar calibration algorithm to realize the camera calibration. Experiments show that this method is of higher accuracy and efficiency, and is of stronger practical value.
\end{abstract}

\section{Introduction}

With the enhance of the revolution of the CCD imaging and the speed of the image disposing, camera calibration and image distortion correction are population in the photogrammetry and vision detection field. Camera calibration is the key step of the regain the three-dimensional information from the image, and decides the measurement accuracy and efficiency in the vision system. Therefore it is of more significance to research the camera calibration method.

At present the more popular method are two-step method of Tsai [1], improved method of Weng[2], and the plane calibration of Zhang[3]. Because that Zhang's plane calibration is easy to use and make the calibration target, and of high accuracy and stability, it is extensively used. But there arithmetic all supposes that the corresponding relation between the points of the different calibration images is known. And they pay little attention to the matching of the points, and extract the boundary points by manual generally. This will increase the workload of the calibration and the calibration error. Therefore it will be of large significance to calibrate the camera automatically.

According to the requirement of the shooting and target making, there are some automatic calibration methods. But they are complex and interfered by the noise and need to add specific marker on the target and the ranking methods are complex[4-7].

We proposed a fast and easy method of automatic camera calibration on the traditional calibration target. First of all, we designed a home-made filled circular maker box and three circles of different size on the traditional target, and segment the target and detected the boundary from the images by the self-adaption area threshold method. And then detected the edge of the target circles by canny algorithm, and employed the least square method to match the center. Finally we ranked the center dot matrix to match the 3D coordinate based on the principle of cross ratio invariability, and then provided the results as the input of the Zhang's coplanar calibration algorithm to realize the camera calibration. Experiments show that this method is of higher accuracy and efficiency, and is of stronger practical value. The experiment result shows that: the automatic calibration method shortens the calibration time and improves the automation and accuracy and stability.

\section{Traditional calibration method}

Camera calibration is to gain the camera intrinsic parameters and the external parameters of the conversions between the camera and target by the maths conversion and optimizing method on the 
image model. And the imaging model stands the relation of the spatial point and the pixel coordinate, and involves four coordinate systems, such as World coordinate $\operatorname{system}\left(O_{w}, X_{w}, Y_{w}, Z_{w}\right)$, Camera coordinate system $\left(O_{c}, X_{c}, Y_{c}, Z_{c}\right)$, image plane coordinate system $(o, x, y)$, and pixel coordinate $\operatorname{system}\left(o_{1}, u, v\right)$. The conversion between four coordinate systems is as shown in figure 1 :

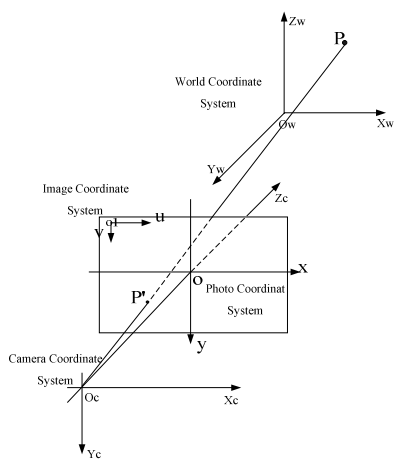

Fig. 1 The coordinate systems conversion

Assuming that point $\mathrm{P}\left(O_{w}, X_{w}, Y_{w}, Z_{w}\right)$ is in the World coordinate system, we can gain the finally pixel coordinate on the imaging model by the formula (1).

$$
Z_{c}\left[\begin{array}{c}
X_{r} \\
y_{r} \\
1
\end{array}\right]=\left[\begin{array}{cccc}
f_{x} / d_{x} & 0 & u_{0} & 0 \\
0 & f_{y} / d_{y} & v_{0} & 0 \\
0 & 0 & 1 & 0
\end{array}\right]\left[\begin{array}{cc}
\boldsymbol{R} & t \\
0 & 1
\end{array}\right]\left[\begin{array}{c}
X_{w} \\
Y_{w} \\
Z_{w} \\
1
\end{array}\right]=\boldsymbol{M}\left[\begin{array}{l}
X_{w} \\
Y_{w} \\
Z_{w} \\
1
\end{array}\right]
$$

There, $f_{x} 、 f_{y}$ is focal length of the level and vertical orientation, $d_{x}, d_{y}$ is the physics size of the level and vertical orientation, $u_{0} 、 v_{0}$ is the pixel coordinate of principal point; The second matrix is the external parameters which describes the conversions between the World coordinate system and the Camera coordinate system.

Given that the pinhole camera model considers no the distortion of the scene, we add in the distortion components.

$$
\begin{aligned}
& \left\{\begin{array}{l}
x_{d}=x_{r}+\Delta x \\
y_{d}=y_{r}+\Delta y
\end{array}\right. \\
& \left\{\begin{array}{l}
\Delta x=x_{r}\left[k_{1}\left(x_{r}^{2}+y_{r}^{2}\right)+k_{2}\left(x_{r}^{2}+y_{r}^{2}\right)^{2}\right]+p_{1}\left(3 x_{r}^{2}+y_{r}^{2}\right)+2 p_{2} x_{r} y_{r} \\
\Delta y=y_{r}\left[k_{1}\left(x_{r}^{2}+y_{r}^{2}\right)+k_{2}\left(x_{r}^{2}+y_{r}^{2}\right)^{2}\right]+p_{2}\left(x_{r}^{2}+3 y_{r}^{2}\right)+2 p_{1} x_{r} y_{r}
\end{array}\right.
\end{aligned}
$$

$x_{d} 、 y_{d}$ is the veritable pixel coordinate; $k_{1} 、 k_{2}$ is the distortion coefficient of radial direction, $p_{1} 、 p_{2}$ is the distortion coefficient of tangential direction.

Traditional calibration methods based the calibration target, such as the MATLAB calibration toolbox, use the target of checkerboard and fix the camera and collect the target images of different position and gesture. And then extract the boundary by manual confirm, as shown in figure 2:

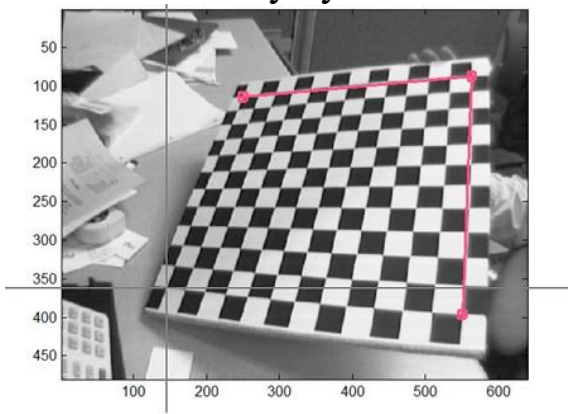

Fig. 2 The traditional extraction of the boundary manually

Utilize the Harris method to get other corner point, and calculate the intrinsic and external parameters by the formula (1) and (3). We can find that there is large error in the process of extracting 
the boundary points, and work load of the whole calibration is large, the calibration efficiency and accuracy are low. Therefore we proposed a fast and easy method of automatic camera calibration.

\section{Automatic Camera Calibration}

In order to calibrate the camera automatically, we need to solve the four problems as follows shown: the target automatically segmentation, gain the boundary automatically, extracting the center coordinate and ranking the center dot matrix coordinate. Arming at the problems, the method is as follows:

1) Use the self-adaption area threshold method to divide the target from the complex background;

2) Exploit the pixel amount of boundary circles automatically gain the boundary;

3) Detect the edge of the target circle by canny algorithm and fit the center by the least square method;

4) According to the principle of cross ratio invariability, use the boundary point coordinate to calculate the row gradient, and then set a self-adaption threshold to matching the points in the same row and rank them by the distance.

\section{Make the calibration target and collect the image}

The traditional checkerboard needs higher quality of the image to ensure the accuracy of the corner extracting. On the contrary, the solid flat disc target needn't high quality. Therefore the final target is as described: $8 \times 8$ circular arrays, interval is $30 \mathrm{~mm}$, the radius of the boundary circles successively is $4 \mathrm{~mm}, 12 \mathrm{~mm}, 15 \mathrm{~mm}$ according to the upper left, left lower and upper right, others are $8 \mathrm{~mm}$, and the outer frame is $10 \mathrm{~mm}$.

The type of the camera is GZL_CL_41C6M_C, and the focal length is $16 \sim 25 \mathrm{~mm}$, and the physics size of pixel is $5.5 \mathrm{um} \times 5.5 \mathrm{um}$. Fix the camera and rotate the target, and collect 12 calibration images, and then save.

\section{Segment the target automatically}

Because that the background in the calibration image will affect the detecting and extracting the point coordinate, we must segment the target from the image automatically so as to calibrate the camera automatically.

Therefore this paper proposed a automatic segmentation arithmetic based on the area and rectangularity(which is the ratio of the number of the area and the Minimum Bounding Rectangle, as shown in formula 5) of the threshold. First of all, detect the edge information and the topology relation. And then reject the noise based on the area and the rectangularity. Finally only extract the position of the interval in the original image. Therefore $r=n_{\text {area }} /(a * b), n_{\text {area }}$ is the number of the pixel in the link area; $a, b$ successively is the length and wide of the Minimum Bounding Rectangle.

\section{Detect the boundary points automatically}

Detect the link area on the target which was divided from the complex background in section 2.2, and record the area pixels which as ranked. According the target and the circles size, we can treat the smallest, the largest and the second largest circle.

\section{Extract the center automatically}

Cut the circle according to link area detected in section 2.3, and detect the edge of the circle by use of canny arithmetic to get all the edges of the circles. Finally fit the center by the improved least square method to gain all the point coordinates. The detail improved least square method is as shown bellows:

1) Extract $m$ points in the edge pixel of the circle, and use the least square method to fit the parameters of the ellipse;

2) Calculate the center based on the parameters and the error between every edge pixel coordinate and the ellipse;

3) Set a suitable threshold and sort the edge pixel as inliters (which is good to the ellipse relatively) and outliters (which is bad to the ellipse relatively), and record the confidence times of every pixel;

4) Repeat the step 2 and $3 \mathrm{n}$ times, and select edge pixel which is in high confidence, named set $U$; 
5) Fit the final parameters based on the edge pixel in set $U$ and calculate the center;

\section{Rank the points automatically}

It is a must to rank the points in order to calibrate automatically. According to the principle of cross ratio invariability, set the smallest boundary circle as origin, named $A$. And then calculate the row gradient, and then set a self-adaption threshold to matching the points in the same row and rank them by the distance. The detail steps are as follows:

1) Assuming the number of the circle on the target is $m \times n$, and the boundary circle is successively $A, B$ and $C$ according to the radius (the upper left $A$, left lower $B$ and upper right $C$ ),;

2) Detect the boundary circles and fit the centers, named $A\left(u_{1}, v_{1}\right), B\left(u_{2}, v_{2}\right), C\left(u_{3}, v_{3}\right)$;

3) Calculate the gradient of line $A C$ and name it as $k_{1}$;

$k_{1}=\frac{v_{3}-v_{1}}{u_{3}-u_{1}}$

4) Calculate the equation of line $A B$ by use of the coordinate of $A$ and $B$. And then calculate the distance between all the points and the line $A B$ named $D_{i}$, and set a self-adaption threshold $\varepsilon$ to extract the points on line $A B$ and rank them according the distance to $A$;

$$
\begin{aligned}
& v_{i}=k_{2} *\left(u_{i}-u_{1}\right)+v_{1} \\
& D_{i}=\frac{k_{2} *\left(u_{i}-u_{1}\right)-v_{i}+v_{1}}{\sqrt{k_{2}^{2}+1}}
\end{aligned}
$$

Where $k_{2}=\left(v_{2}-v_{1}\right) /\left(u_{2}-u_{1}\right)$.

5) Set $A, B_{2}, \ldots, B_{m-1}, B$ successively as the fixed point and calculate the line equation with the gradient $\mathrm{k} 1$. And then extract the points on the line and rank them according the distance to the fixed point named $B_{i j}$. Take $B_{i}$ as example, then the line equation is

$v=k_{1} *\left(u-u u_{i}\right)+v v_{i}$

6) Then calculate the distance from all the points to the line, and extract the points on the line, and rank them according the distance. Assuming that some point is located on the line and the order is $j$, therefore the order on the whole target is

$$
N_{i j}=n *(i-1)+j
$$
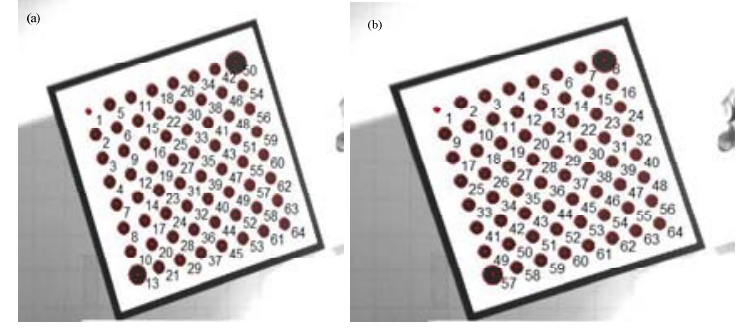

Fig. 3 The comparison of ranked and unranked points (a-unranked, b-ranked)

\section{The result of experiment}

Treat the ranked circle center coordinate as the input of the Zhang calibration arithmetic, and then calculate the parameters of camera and the re-projection error (the Euclidean distance between the projection point and the measurement point). Calibrate the camera respectively by the traditional arithmetic and the automatic arithmetic, and the results are shown in table 1, and the re-projection error is shown in figure 4.

Table 1 Calibration result of the traditional and automatic arithmetic

\begin{tabular}{lcccccccccc}
\hline & \multicolumn{4}{c}{ Camera Parmeters } & \multicolumn{3}{c}{ Distortion Coefficient } & \multirow{2}{*}{ re-projection error } & time \\
\cline { 2 - 8 } & $f_{x}$ & $f_{y}$ & $u_{0}$ & $v_{0}$ & $k_{1}$ & $k_{2}$ & $p_{2}$ & $p_{1}$ & & \\
\hline $\begin{array}{l}\text { Traditional } \\
\text { arithmetic }\end{array}$ & 15.653 & 15.736 & 521.23 & 620.32 & -0.3762 & 0.4335 & -0.0483 & -0.0276 & $(0.4255,0.4652)$ & $30 \mathrm{~s}$ \\
$\begin{array}{c}\text { automatic } \\
\text { arithmetic }\end{array}$ & 15.902 & 15.831 & 526.16 & 624.98 & -0.2871 & 0.3732 & -0.0430 & -0.0241 & $(0.1274,0.1927)$ & $200 \mathrm{~s}$ \\
\hline
\end{tabular}


In table 1 , we can find that the automatic calibration arithmetic need only 30 seconds to complete the calibration and the calibration speed is largely enhanced compared with the traditional arithmetic. And the average re-projection error is smaller.

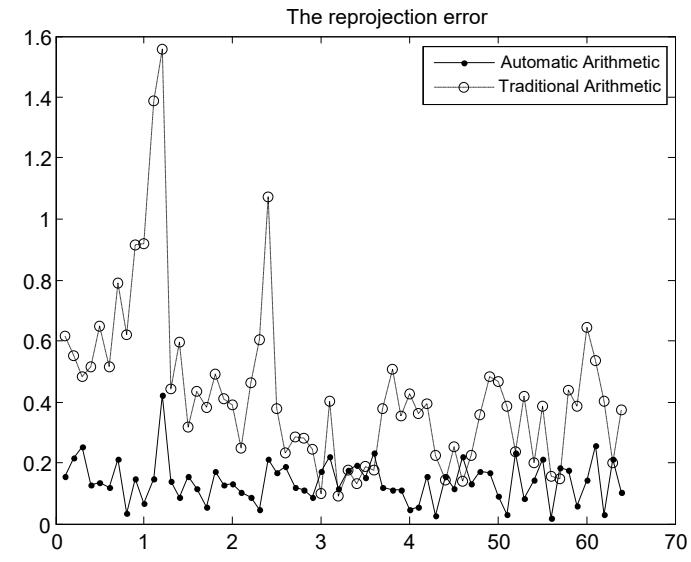

Fig. 4 The re-projection error

In figure 4, we can find that the range of the re-projection error by the automatic arithmetic is smaller than the traditional calibration arithmetic, and is of better stability.

\section{Summary}

It is beautiful to solve the problems of target segmentation and boundary ensure by adding the outer frame and boundary circles of different size on the traditional target. And this paper proposed the automatically ranking arithmetic according to the principle of cross ratio invariability. It is easy and fast to calibrate the camera with nobody operation. The experiment result shows that, it is of high speed, accuracy and stability to calibrate camera, and is very suitable to the project apply situation.

\section{References}

[1] R. Y. Tsai. A versatile camera calibration technique for high-accuracy 3D machine vision metrology using off-the-self TV cameras and lens[J]. IEEE J. Robotics and Automation, 1987, 3(4): 323-344

[2] J. Weng, P. Cohen, M. Herniou et al. Camera calibration with distortion models and accuracy evaluation [J]. IEEE Trans. Pattern Analysis and Machine Intelligence, 1992, 14(10): 965- 980

[3] Zhang. Camera calibration with one-dimensional objects[J]. IEEE Trans. Pattern Analysis Intelligence, 2004, 26(7): 892-899

[4] Xu Yong, Guo Pengyu, Long Gucan, et al. Research on fully automatic camera calibration method[J]. Acta Optica Sinica, 2013, 33(6), 0612007

[5] Bu Penghui, Zhao Hong, Gu Feifei, et al. A fully automatic camera calibration method based on planar target[J]. Acta Optica Sinica,2014, 34(10): 1015004

[6] M. Friel , C. Hughes, P. Denny et al. Automatic calibration of fish-eye cameras from automotive video sequences[J]. IET Intell. Transp. Syst, 2010, 4(2): 136- 148

[7] Zhao Bin, Zhou Jun, Automatic detection and soring of corners by improve chessboard pattern[J]. Optics and Precision Engineering, 2015, 23(1) :237-244 